\title{
Evaluation of the Efficiency of Genome Editing Tools by a Frameshift Fluorescence Protein Reporter
}

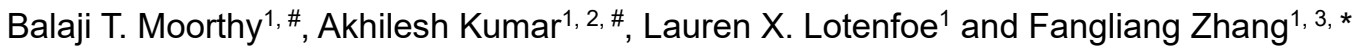

${ }^{1}$ Dept. of Molecular and Cellular Pharmacology, Miller School of Medicine, University of Miami, FL 33136, USA; ${ }^{2}$ Dept. of Botany, Banaras Hindu University, Varanasi, India; ${ }^{3}$ Sylvester comprehensive cancer center, University of Miami, FL 33136, USA

*Corresponding author: fzhang2@miami.edu

\#Contributed equally to this work

[Abstract] In the last decade, genome editing has been the center of attention as a novel tool for mechanistic investigations and for potential clinical applications. Various genome editing tools like meganucleases, zinc finger nucleases (ZFNs), transcription activator-like effector-based nucleases (TALEN), and the clustered regularly interspaced short palindromic repeats (CRISPR)-associated genes (Cas), have been developed in recent years. For the optimal use as well as continued developments of these genome editing tools, the evaluation of their efficiencies and accuracies is vital. Here, we present a protocol for a reporter based on frameshift fluorescence protein which we recently developed to evaluate the efficiency and accuracy of genome editing tools. In this method, a $\sim 20$ bp target sequence containing frame-shifting is inserted after the start codon of a cerulean fluorescence protein (CFP) to inactivate its fluorescence, and only a new insertion/deletion event in the target sequence will reactivate the CFP fluorescence. To increase the traceability, an internal ribosome entry site and a red fluorescence protein, mCherryFP, are placed downstream of the reporter. The percentage of CFP-positive cells resulted from in/del mediated fluorescence restoration can be quantified by fluorescence measuring devices as the readout for genome editing frequency. As a demonstration, we present the usage for CRISPR-Cas9 technique here with flow cytometer as the readout for fluorescence changes.

Keywords: Insertion-deletion, In-del, Reporter, CRIPSR-Cas9, Genome editing, NHEJ

[Background] Genome editing tools are very important for the investigations of biological mechanisms and prevention and/or treatment of genetic diseases (Maeder and Gersbach, 2016). In the last couple of decades, several genome editing tools have been introduced, which include the meganucleases (Epinat et al., 2003), zinc finger nucleases (ZFNs) (Kim et al., 1996), transcription activator-like effectorbased nucleases (TALEN) (Christian et al., 2010), and the clustered regularly interspaced short palindromic repeats (CRISPR)-associated genes (Cas) (Jinek et al., 2012; Cong et al., 2013; Sander and Joung, 2014). In general, these tools create DNA double stranded breaks (DSB) to trigger genome editing in vivo (Maeder and Gersbach, 2016). The evaluation of the efficiencies and specificities of genome editing tool is essential for their applications and further developments. In our recent published study, we described a reporter that can generate quantitative readout for genome editing efficiency (Kumar et al., 2019). In this system, a 20 bp target sequence is placed in a multiple cloning site (MCS) 
which is right after the start codon of Cerulean fluorescence protein (CFP) to generate a frame-shift of the open reading frame (ORF). This frameshifted-CFP (FsCFP) can be used as a reporter of genome editing because only when there is a successful DNA-double strand break (DSB) event on the target sequence followed with a non-homologous end joining (NHEJ) to generate an in/del event to shift the reading frame to a correct order (by a chance of up to 1/3), the CFP fluorescence will be reactivated as a positive readout. To facilitate the quantification, an internal ribosome entry site (IRES) and a red fluorescence protein, mCherryFP, is placed after the reporter. In principle, this reporter can be applied to any genome editing system as long as a DSB and NHEJ are expected from the editing. This approach can effectively detect low-efficiency editing in a population of cells with very low false negative or false positive. Furthermore, in this method, the positive cells can be conveniently identified and enriched for the examination or validation of the in/del event in the genome. Also, this method can be easily adapted for screening to optimize the genome-editing enzyme or the other components (such as guide-RNA) in the positive cells. Here, we used the CRISPR-Cas9 technique as a demonstration and the flow cytometry as the readout of the fluorescence events.

In this protocol, the target sequence is inserted between restriction sites of Notl and Xhol before CFP reporter together with sequence for the optimal recognition of Cas9 and a premature STOP codon to create a frame shift. The reporter region is then integrated in the nuclear genome of the target cell by the assistance of lentivirus. The target cells expressing the red fluorescence protein are then isolated by fluorescence-activated cell sorting (FACS), before vectors containing the Cas9 and gRNA are introduced into these cells. After incubation, the ratio of the CFP over mCherryFP was measured in flow cytometry to provide quantitative measurement for the efficiency of the genome editing.

\section{Materials and Reagents}

\section{Materials}

1. Cell Culture dish $150 \times 25 \mathrm{~mm}$ (Asi, catalog number: TD0150)

2. Cell Culture dish $90 \times 20 \mathrm{~mm}$ (Asi, catalog number: TD0100)

3. $5 \mathrm{ml}$ serological pipet (Asi, catalog number: SP205)

4. $10 \mathrm{ml}$ serological pipet (Asi, catalog number: SP210)

5. Cell Culture Flask $75 \mathrm{~cm}^{2}$, filter cap (Asi, catalog number: TV0075)

6. Cell Culture Flask $25 \mathrm{~cm}^{2}$, filter cap (Asi, catalog number: TV0025)

7. Syringe filter, PES $25 \mathrm{~mm}, 0.45 \mu \mathrm{m}$ (Asi, catalog number: TE45-5)

8. $10 \mathrm{ml}$ syringes (BD, catalog number: 309604 )

9. $15 \mathrm{ml}$ and $50 \mathrm{ml}$ conical tubes (Denville, catalog number: C1062-P)

10. $18 \mathrm{G} \times 1 \frac{1}{2}$ needles (BD, catalog number: 305196 )

11. Bottle top filtration-2 $\mu \mathrm{m}$ PES (VWR, catalog number: 97066-202)

12. $15 \mathrm{ml}$ centrifuge tubes (CellPro, catalog number: CN5600)

13. $50 \mathrm{ml}$ centrifuge tubes (CellPro, catalog number: CN5603)

14. Filter Pipet Tips $1,250 \mu \mathrm{l}$ (TruPoint, catalog number: FT1250) 
15. Filter Pipet Tips $200 \mu \mathrm{l}$ (TruPoint, catalog number: FT1200)

16. Filter Pipet Tips $20 \mu l$ (TruPoint, catalog number: FT1020)

17. Filter Pipet Tips $10 \mu \mathrm{l}$ (TruPoint, catalog number: FT1010)

18. Falcon FACS tubes with $35 \mu$ Mcell strainer (BD, catalog number: 352235 )

19. Falcon FACS collection tubes (BD, catalog number: 352063 )

20. Ice buckets

21. T4 DNA ligase (New England Biolabs, catalog number: M0202S)

22. Gel extraction kit (EZ, catalog number: M1002-50)

23. One Shot ${ }^{\mathrm{TM}}$ TOP10 Chemically Competent E. coli (Invitrogen, catalog number: C404003)

24. ZymoPURE ${ }^{\text {TM }}$ II Plasmid Midiprep Kit (Genesee Scientific, catalog number: 11-550B)

25. QIAprep Spin Miniprep Kit (50) (QIAGEN, catalog number: 27104)

\section{Cells and Plasmids}

1. Human embryonic kidney cells (HEK 293T, clone 17) (ATCC, catalog number: CRL-11268)

2. Plasmid PQC-XIG (Addgene, catalog number: w497-1), deposited by Dr. Eric Campeau, who is currently at Zenith Epigenetics Ltd, Calgary, Canada

3. Plasmid pCMV-Delta R8.2 (Addgene, catalog number: 12263), deposited by Dr. Didier Trono at EPFL

4. Plasmid pCMV-VSV-G (Addgene, catalog number: 8454), deposited by Dr. Robert Weinberg at MIT

5. gRNAs custom ordered from Vector builder (https://en.vectorbuilder.com/)

\section{Reagents}

1. High Glucose DMEM (1x) (Life Technologies, catalog number: 11995-065)

2. F-10 media (1x) (Life Technologies, catalog number: 11550-043)

3. $0.25 \%$ Trypsin-EDTA (1x) (Life technologies, catalog number: 25200-072)

4. $10 \%$ FBS (Hyclone, catalog number: SH30910.03)

5. Polybrene Transfection Reagent (Millipore, catalog number: TR-1003-G)

6. Antibiotic-Antimycotic (100x) (Life Technologies, catalog number: 15240062)

7. Polyethylenimine (PEI) (Sigma-Aldrich, catalog number: 408727)

8. T4-ligase Buffer (New England Biolabs, containing $50 \mathrm{mM}$ Tris- $\mathrm{HCl}, 10 \mathrm{mM} \mathrm{MgCl} 2,1 \mathrm{mM}$ ATP, $10 \mathrm{mM}$ DTT, $\mathrm{pH}$ 7.5)

9. DMEM+F10 culture media ( $45 \%$ DMEM $+45 \% \mathrm{~F}-10+10 \% \mathrm{FBS}$, with $1 \times$ Antibiotic-Antimycotic)

10. Nucleotide oligos/primers: 
Table 1. List of primers used in this protocol

\begin{tabular}{|c|c|c|}
\hline Purpose & Name & Sequence $\left(5^{\prime}-3^{\prime}\right)$ \\
\hline \multirow{2}{*}{$\begin{array}{l}\text { Target } \\
\text { Sequence for } \\
\text { the reporter }\end{array}$} & Top-strand target oligo & $\begin{array}{l}\text { ggccgcCATATGTGGGTGGGGGGAGTTT } \\
\text { GCTCCAGGTGAAc }\end{array}$ \\
\hline & Bottom-strand target oligo & $\begin{array}{l}\text { tcgagTTCACCTGGAGCAAACTCCCCCC } \\
\text { ACCCACATATGGcg }\end{array}$ \\
\hline \multirow{4}{*}{$\begin{array}{l}\text { Sequencing } \\
\text { primers for the } \\
\text { reporter vector }\end{array}$} & $\begin{array}{l}\text { Forward primer targeting } \\
\text { CMV promoter }\end{array}$ & AGAGCTCGTTTAGTGAACCGTC \\
\hline & $\begin{array}{l}\text { Reverse primer targeting } \\
\text { IRES }\end{array}$ & $\begin{array}{l}\text { GACGGCAATATGGTGGAAAATAACATAT } \\
\text { AGACAAACGCACACCGG }\end{array}$ \\
\hline & $\begin{array}{l}\text { Forward primer targeting the } \\
\text { border between the CMV } \\
\text { promoter and the cloning } \\
\text { sites }\end{array}$ & $\begin{array}{l}\text { GAGCTCGTTTAGTGAACCGTCAGATCG } \\
\text { CCTGGAGACGCCATCCACG }\end{array}$ \\
\hline & $\begin{array}{l}\text { Reverse primer specifically } \\
\text { targeting CFP: }\end{array}$ & $\begin{array}{l}\text { TAGTTGCCGTCGTCCTTGAAGAAGATG } \\
\text { GTGCGCTCCTGGACGTAGCC }\end{array}$ \\
\hline
\end{tabular}

\section{Equipment}

1. Centrifuge $5424 \mathrm{R}$ (Eppendorf, catalog number: 540400138 )

2. Labnet Accublock Digital Dry Bath (Labnet, catalog number:19-41620)

3. Gene Mote Vortex Mixer (Bioexpress, catalog number: S-3200-1)

4. $\mathrm{CO}_{2}$ incubator MCO-19AIC (UV) (Panasonic, catalog number: 13010002)

5. Centrifuge 5702 (Eppendorf, catalog number: 022626205)

6. Fluorescent microscope

7. Aria-IIU flow cytometer (BD)

\section{Software}

1. FCS Express 6 (Denovo software-https://denovosoftware.com/)

\section{Procedure}

A. Generation of reporter and gRNA constructs

1. Construction of the Frameshift(Fs) CFP-mCherryFP reporter

a. The nucleotide sequence consisting of CFP, IRES and mCherryFP and flanked with Notl and EcoRV restriction sites was synthesized by using the service of Genscript, NJ. See Figure 1 for the illustration of the vector map and the nucleotide sequence. 
Note: The CFP was chosen as the frameshift reporter due to its lack of internal starting codons (ATG) near the 5 '-end, which is essential to prevent the generation of smaller proteins that are potentially still fluorescent (such as in the case of mCherryFP). Although GFP also lacks internal ATG near the 5'-end, it is often used as a marker in many vectors containing the genome editing machineries. In addition to fluorescence proteins, proteins that don't have internal ATG near the 5'-end and can be specifically detected or selected may also be designed as frameshifting reporters. These may include luciferases, $\beta$ galactosidase, or aminoglycoside 3'-phosphotransferase.

A

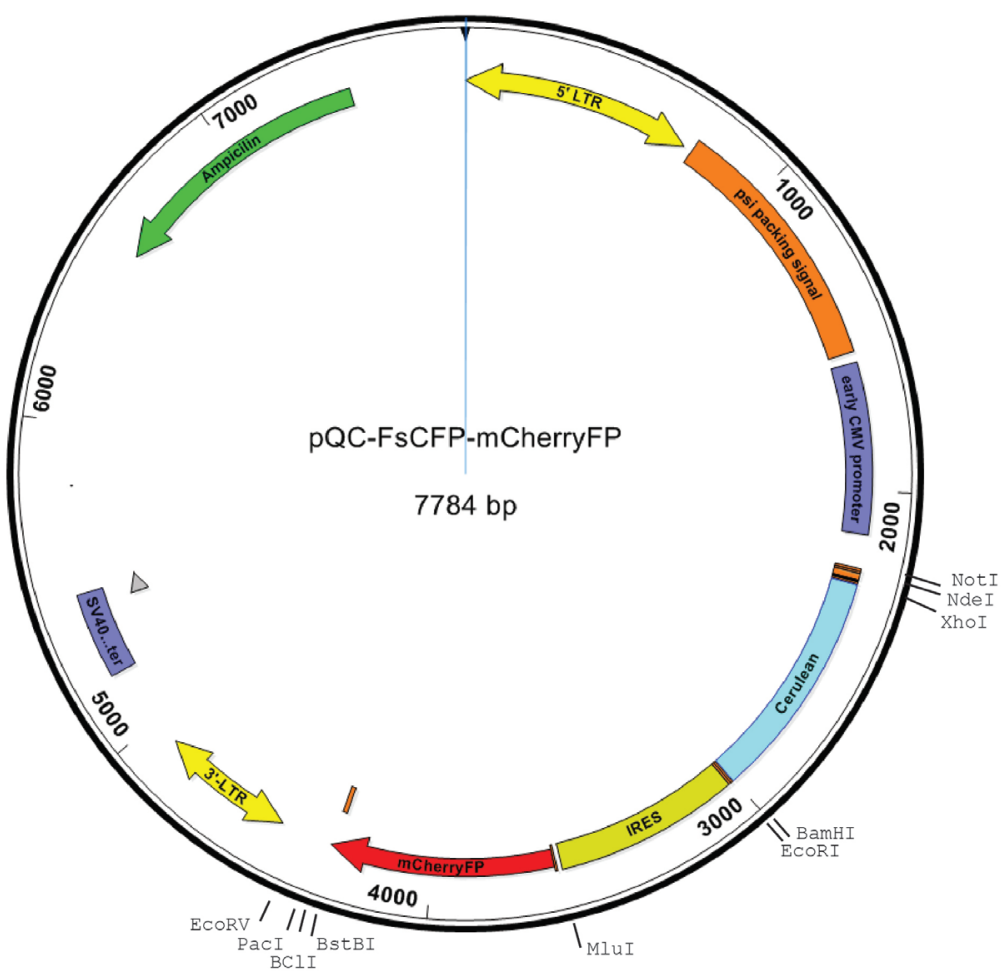

B
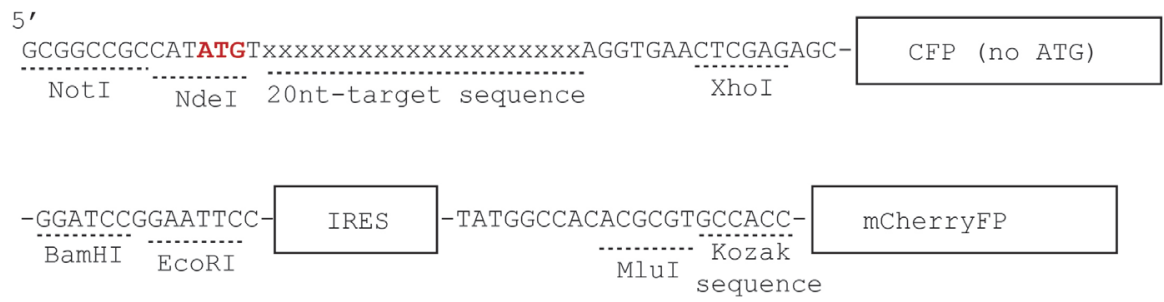

-TTCGAATGATCAATTAATTAAGAATTATCAAGCTTATCGATACCGTCGAGATATC
BstBI $\cdots . . . . .$.
BClI

Figure 1. Construction of the reporter plasmids. In (A), there is a schematic representation of the vector map of pQC-FSCFP-mCherryFP, which is modified from PQC-XIG vector (from Addgene) with a synthesized nucleotide sequence as shown in (B). The synthesized sequence (5'-strand shown) is to be cloned into the PQC-XIG vector by restriction sites Notl and EcoRV. 
This cloning will replace the original IRES and GFP regions. It will also introduce the CFP coding sequence and additional cloning sites. In the demonstrated example here it also included the $20 \mathrm{nt}$ target sequence.

b. The above-described nucleotide sequence is cloned into a template vector, PQC-XIG, using the restriction sites Notl and EcoRV ( $5^{\prime}$ end and $3^{\prime}$ end, respectively).

Note: This replaces the original IRES and GFP sequences in the $p Q C-X I G$ vector for the sake of introducing multiple cloning sites flanking the CFP and mCherryFP sequences for future cloning purposes.

c. Digest the FsCFP-mCherryFP reporter plasmid generated from above with Not1 and Xho1 (5' end and 3' end respectively). Gel purify the digested vector using Gel extraction kit (EZ) and store the product at $-20^{\circ} \mathrm{C}$.

Note: Do not perform phosphatase treatment during or after the digestion, unless the custom ordered oligos were 3'-phosphorylated.

2. Introducing the target sequence in FsCFP-mCherryFP reporter (see also Table 1 for the nucleotide sequences of primer/oligos)

a. Synthesizing the target sequence-For example, the target sequence for VEGF-A gene in human is 5'-GGGTGGGGGGAGTTTGCTCC-3'. The targeting sequence is placed after the START codon, followed by a protospacer adjacent motif (PAM) site and a premature STOP codon. If necessary, a few (1-5) extra nucleotides can be added to ensure that the CFP is out of frame. In the shown example, the inclusion of a premature STOP codon is to prevent the production of a long-length protein resulting from the frameshifted coding sequence of CFP, which may be harmful to the cell (Figure 2). The nucleotide oligos (both 5' and 3' strand) containing the above (target) sequence as well as overhanging nucleotides which would result from Notl $\left(5^{\prime}\right)$ and Xhol $\left(3^{\prime}\right)$ digestion were then synthesized by using the service of Genscript, NJ:

Top-strand target oligo: 5'-ggccgcCATATGTGGGTGGGGGGAGTTTGCTCCAGGTGAAc-3' Bottom-strand target oligo: 5'tcgagTTCACCTGGAGCAAACTCCCCCCACCCACATATGGcg-3'

Notes: The two oligos, if annealed, represent the double-digestion product of Notl (5') and Xhol (3'). (Figure 3)

Detailed nucleotide sequence around the target insert site

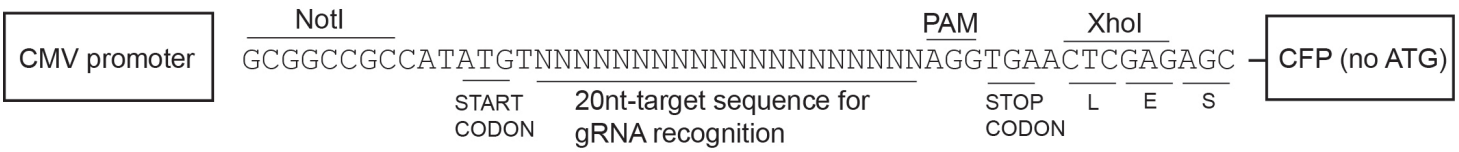

Figure 2. Schematic representation of the nucleotide sequence flanking the target insert region. The position of the 20-nucleotide (nt) gRNA-matching site, the 3nt-protospacer adjacent motif (PAM) sequence for Cas9 binding is indicated along with the START and STOP codon. 
The two extra nucleotides in this particular example are included to ensure the out-of-frame CFP. The premature STOP codon is included to prevent the translation of a long-length product from the START codon on the shifted frame of the CFP coding sequence.

Top strand oligo $\quad 5^{\prime}$-ggccgCCATATGTGGGTGGGGGGAGTTTGCTCCAGGTGAAC-3'

Bottom strand oligo 5' -tcgagTTCACCTGGAGCAAACTCCCCCCACCCACATATGGCg-3'

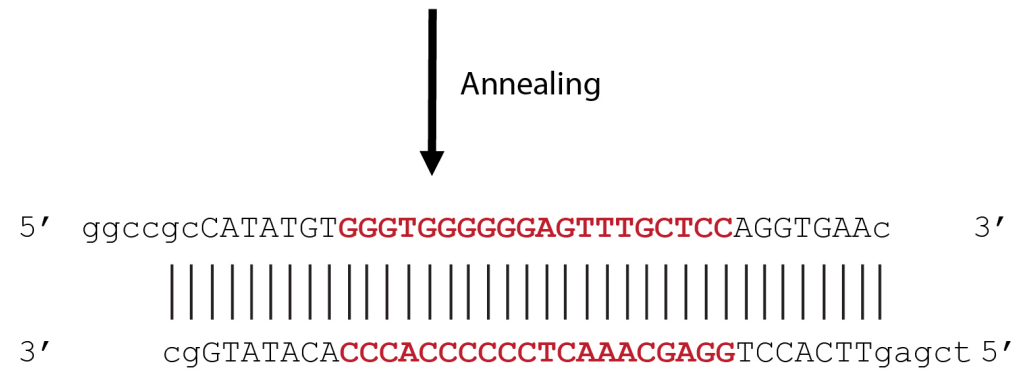

Figure 3. Annealing of 5'-strand and 3'-strand oligos. The oligo sequences containing an example with 20-nt target sequence (in bold red font) derived from human VEGF gene, the START codon, PAM site, and premature STOP codon, as well as overhanging nucleotides which would result from Notl $\left(5^{\prime}\right)$ and Xhol $\left(3^{\prime}\right)$ digestion (shown in lower case). After annealing, the $5^{\prime}$ and 3 ' strands together represent the digested product of Not1 and Xho1.

b. Annealing-In an Eppendorf tube, mix the two oligos from above (Step A2a) in 1:1 ratio to a final concentration of $20 \mu \mathrm{M}$ (each) in 1x T4 ligase buffer. Place the tube in the boiling water $\left(100{ }^{\circ} \mathrm{C}\right.$ ) for $10-20 \mathrm{~min}$ and allow the water to cool down at room temperature for $8-12 \mathrm{~h}$ for the annealing process. The product can then be used freshly or stored at $-20^{\circ} \mathrm{C}$.

c. Ligation-Mix the annealed oligos with the digested vector from Step A1c. The oligo would be 3-10 times (5 time is recommended) in excess compared to the vector in molecules. Ligation is carried out by the addition of T4 DNA ligase (NEB) in the presence of 1x T4 ligase buffer (NEB). For each $20 \mu \mathrm{l}$ reaction which typically contains $1 \mu \mathrm{g}$ of digested vector and $0.05 \mu \mathrm{g}$ of annealed oligos, $1 \mu \mathrm{l}$ of ligase was added. The ligation reaction is performed at $16{ }^{\circ} \mathrm{C}$ for $2 \mathrm{~h}$ followed by $8{ }^{\circ} \mathrm{C}$ overnight

d. Bacterial transformation-The ligated product from above is introduced into Top10 cells (Invitrogen), according to the manufacturer's instructions. In general, 2 3 $\mu \mathrm{l}$ of the ligation product was used to transform $50 \mu \mathrm{l}$ of Bacteria. The transformed bacteria is plated on to LB + Ampicillin $(50 \mu \mathrm{g} / \mathrm{ml})$ plates for incubation at $37{ }^{\circ} \mathrm{C}$ overnight. Grown colonies are picked from the plate to grow in $5 \mathrm{ml}$ liquid LB + Ampicillin $(50 \mu \mathrm{g} / \mathrm{ml})$ overnight. Plasmid is then isolated using Qiagen QiaPrep Spin Miniprep kit and then submitted for Sanger sequencing with primers specifically targeting the CMV promoter, the CFP region (without cross-reacting with the mCherryFP region), and the IRES region of the plasmid (Figure 1A). Primers used in this protocol are listed in Table 1. 
Forward primer targeting CMV promoter:

\section{5'-AGAGCTCGTTTAGTGAACCGTC-3'}

Reverse primer targeting IRES:

\section{5'-GACGGCAATATGGTGGAAAATAACATATAGACAAACGCACACCGG-3'}

Forward primer targeting the border between the CMV promoter and the cloning sites:

5'-GAGCTCGTTTAGTGAACCGTCAGATCGCCTGGAGACGCCATCCACG-3'

Reverse primer specifically targeting CFP:

\section{5'-TAGTTGCCGTCGTCCTTGAAGAAGATGGTGCGCTCCTGGACGTAGCC-3'}

e. Colonies carrying the plasmid containing the expected insertion sequence in the FsCFPmCherryFP reporter is then grown in $100 \mathrm{ml}$ liquid LB + Ampicillin $(50 \mu \mathrm{g} / \mathrm{ml})$ overnight. Plasmid is then isolated with the Midiprep Kit (ZymoPURE ${ }^{T M}$ II Plasmid Midiprep Kit). This product can be stored at $20^{\circ} \mathrm{C}$.

3. Guide RNA (gRNA) construction

The plasmids for gRNA and Cas9 can be designed and purchased from Vector Builder INC (https://en.vectorbuilder.com/), with GFP as a selection marker. For example, the gRNA sequence for VEGF will be -GGGTGGGGGGAGTTTGCTCC. As a control, scramble gRNA should also be ordered.

B. Creation of cell lines with FsCFP-mCherryFP reporter and gRNA

Preparation of cell lines with reporter and gRNA is a 2-step sequential process.

Step B1: Cell line of interest + FsCFP-mCherryFP (reporter plasmid) $\rightarrow$ sort for mCherry positive cells

Step B2: Cell line with FsCFP-mCherryFP + (target) gRNA-GFP $\rightarrow$ sort for mCherry and GFP double positive cells.

\section{Step B1: Generation of cell line with FsCFP-mCherryFP reporter}

1. Production of lentiviral particles in HEK 293T cell line

To begin with, seed the HEK 293 T cells so that they will reach $\sim 75 \%$ confluency in a T-25 culture flask, which can be estimated by microscopic observations. Change the DMEM+ F10 culture just before the transfection steps below.

a. In a $1.5 \mathrm{ml}$ Eppendorf tube (tube 1), add $1 \mu \mathrm{g}$ of VSV-G, $6 \mu \mathrm{g}$ of Delta R8.2, and $6.5 \mu \mathrm{g}$ of reporter plasmid containing the targeting sequence (from Step A2e) to $0.5 \mathrm{ml}$ of DMEM + F10 media. Vortex for 20-30 s for mixing and spin down briefly (less than $10 \mathrm{~s}$ ) in minispinner or in a common desktop centrifuge at 2,000 $\mathrm{xg}$.

b. Add $0.5 \mathrm{ml}$ of DMEM to another Eppendorf tube (tube 2), then add $30 \mu \mathrm{l}$ of activated Polyethylenimine reagent (Sigma) or other types of transfection reagents such as Lipofectamine (Thermo-Fisher) or JetPrime (Polyplus-Transfection). Vortex immediately for $30 \mathrm{~s}$. Spin down the components briefly as above. Add the contents from tube 1 to tube 2. 
Vortex the tube immediately for $20-30 \mathrm{~s}$ and then spin down briefly again. Incubate at room temperature (RT) for 20-30 min.

c. After the incubation, add the transfection mix drop by drop to the flask containing the HEK $293 \mathrm{~T}$ cells and the fresh media. Mix gently and place the flask at $37{ }^{\circ} \mathrm{C}, 5 \% \mathrm{CO}_{2}$ incubator for the production of viral particles in the next three days. The majority of these cells $(>50 \%)$ are expected to express mCherryFP (excitation $587 \mathrm{~nm}$, emission $610 \mathrm{~nm}$ ) after $24 \mathrm{~h}$, which can be visually verified by the presence of red fluorescence under a fluorescence microscope.

d. Harvest the media from the virus-producing HEK $293 \mathrm{~T}$ cells after $24 \mathrm{~h}, 48 \mathrm{~h}$ and $72 \mathrm{~h}$ (if necessary). Replace with fresh culture media.

e. Place the harvested media in a sterile $50 \mathrm{ml}$ conical tube. Add polybrene to the final concentration of $30 \mu \mathrm{g} / \mathrm{ml}$. Vortex briefly to mix.

f. [Optional] Spin down at a swing-bucket rotor at 1,000 $\times \mathrm{g}$ for $2 \mathrm{~min}$ to remove cell debris.

g. Filter the supernatant using syringe and a 0.45 -micrometer syringe filter. The supernatant should be used immediately on the target cell, although it can also be stored at $4{ }^{\circ} \mathrm{C}$ for up to three days. If a longer storage is required, the supernatant should be distributed into aliquots and snap frozen in liquid nitrogen and then stored at $-80^{\circ} \mathrm{C}$.

2. Transduction of target cells

As a demonstration, HEK293T cells are used as target cells to receive the lentivirus produced from above (Procedure B, Step B1-1).

a. Grow HEK 293T cells in T-75 flask. The density of the cells should be under $30 \%$ confluent before the 1st viral transduction, as visually estimated under an optical microscope.

b. Dilute the virus-containing supernatant harvested from the T-25 of cells at Step B1-1 with $2 x$ volume of fresh culture media. Replace the media in the T-75 flask containing the target cells with this mix containing the virus and polybrene (final concentration $10 \mu \mathrm{g} / \mathrm{ml}$ ).

c. Repeat the above step for one more time in the next day.

d. Check the mCherryFP fluorescence under fluorescence microscope to ensure that the fluorescence positive cells are less than $20 \%$, in order to avoid over-infection.

Note: It is important to keep the target cells less than $75 \%$ confluence at any time during the viral induction stage.

3. Sorting of target cells carrying the reporter plasmid

a. Trypsinize and collect the target cells by flushing with fresh culture media (at least 5 volume of the trypsin solution used). Transfer the mix to a sterile $50 \mathrm{ml}$ conical tube. Collect the cells by centrifugation in a swing bucket rotor at $1,500 \times \mathrm{g}$ for $3 \mathrm{~min}$ at room temperature.

Note: Create a backup of these unsorted cells by freezing them in $-80^{\circ} \mathrm{C}$.

b. Resuspend the cells in $1 \mathrm{ml}$ of sorting medium (DMEM + antibiotic-antimycotic), which should generate a density of $\sim 10$ million cells $/ \mathrm{ml}$.

c. Pass the cells through the FACS tubes with 35-micrometer cell strainer.

d. Add $1 \mathrm{ml}$ of sorting medium in the FACS collection tubes. 
e. Use target cells without any mCherry plasmid as negative control for gating purposes in flow cytometry.

f. Sort the cells for mCherry positive using FACS-BD Aria-llu flow cytometer.

Note: For success of subsequent culture, we recommend a minimum of 20,000 cells should be acquired for each sample.

g. Culture the mCherry positive cells in T-25 flask with $5 \mathrm{ml}$ fresh media. Expand the culture to bigger flasks (such as T-75) to generate cells carrying the reporter for the next step and keep aliquots for storages at $-80^{\circ} \mathrm{C}$.

Note: The quality of the cells expressing the reporter should be periodically inspected, which can be verified by the presence of red fluorescence in at least $90 \%$ of the cells under fluorescence microscope.

\section{Step B2: Generation of cell line co-expressing FsCFP-mCherryFP and gRNA-GFP}

4. Packaging of lentiviral particles in HEK 293T cell line

This step is similar to the one mentioned above (Step B1), except that the gRNA-GFP plasmid (the VEGF-gRNA-GFP or the scrambled-sequence gRNA-GFP) will be used with these plasmids VSV-G (coding for viral envelop protein) and DeltaR8.2 (coding for reverse transcriptase HIV1-pol and packaging factor HIV1-gag suitable for lentivirus).

5. Transduction of target cells expressing reporter plasmid with the lentivirus containing gRNAGFP.

Before proceeding, do a quick visual verification under the fluorescence microscope for the target cells expressing reporter plasmid to make sure more than $90 \%$ of cells are mCherrypositive.

Note: The target cells (expressing reporter plasmid) grown in the T-25 flask should be around $30 \%$ confluent right before the 1 st viral induction (24 h).

The target cells can be repeatedly induced for 2-3 days. In the end of the induction, the GFPproducing cells should be less than $50 \%$ of the population to avoid over-induction.

6. Sorting of target cells carrying both the reporter and gRNA

After $72 \mathrm{~h}$, check the transfection efficiency of target cells under fluorescence microscope for the presence of both mCherry and GFP fluorescence.

Note: Create a backup of these unsorted cells by freezing them in $-80^{\circ} \mathrm{C}$.

a. Process the cells for sorting as described above (Steps B3a-B3d)

b. Use appropriate positive controls for gating purposes while sorting. Cells don't express any fluorescence proteins, or expressing GFP-only, mCherryFP-only, or GFP plus mCherryFP should be used as negative controls to set the gates for CFP-positive events. The detection threshold in general can be set at two folds above the maximum signal generated by the negative controls. If necessary, cells expressing functional GFP, mCherryFP, and CFP can also be used as the positive control (although this is usually not necessary). 
c. Acquire a minimum of 100,000 events for the double-positive cells to ensure reliable detection of any triple-positive cells.

d. Sort the target cells for mCherry and GFP double-positive and any mCherryFP, GFP, and CFP triple-positive populations using FACS-BD Aria-llu flow cytometer. The setting of the sorting gates for CFP-positive events should be adjusted so that the collection window is at least by 2 -folds higher than the edge of the main population at the CFP channel from any of the three negative controls. Conversely, the collected double-positive cells should not contain CFP signal higher than that of the negative controls.

e. Grow and expand the double-positive and triple-positive cells for further analysis.

Note: Create a backup of these sorted cells by freezing them at $-80^{\circ} \mathrm{C}$.

7. FACS data analysis

a. Analyze the FACS data by using either FCS Express 6 or FlowJo. The percentage of CFPpositive cells among the double-positive cells can be quantified using FCS Express 6 and MS-Excel.

b. Forward and side scattering should be used to exclude cell fragments/debris or cell clumps/clusters. The threshold for detection of the GFP and mCherryFP signals should be established with parental cells that were either not treated, or stably transduced with GFPonly or mCherryFP-only expression vectors. The gating threshold for the double-positive cells (GFP and mCherryFP) should be at least one fold away from the individual positives (GFP-only and mCherry-only) (Figure 4). 

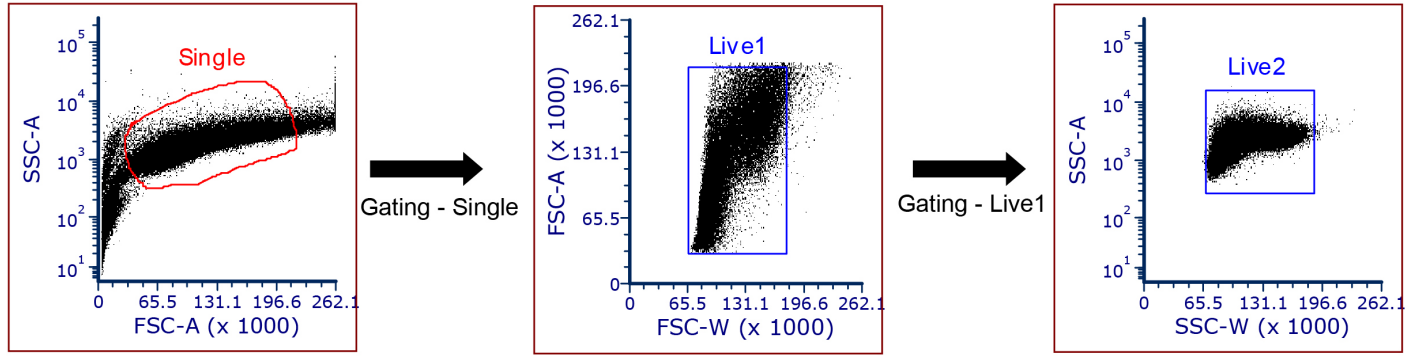

Negative control
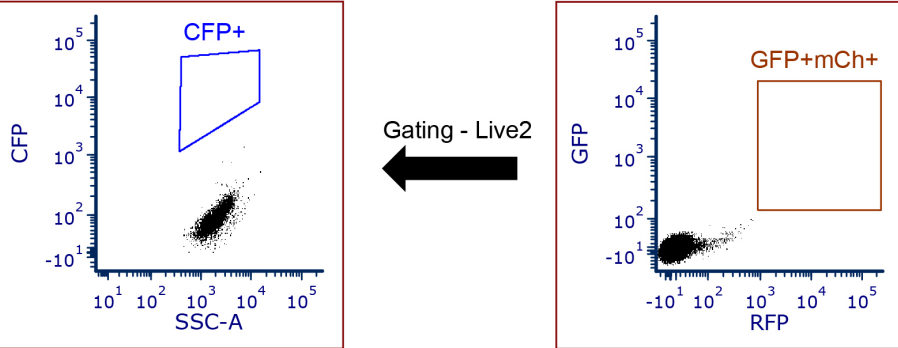

mCherry-only control
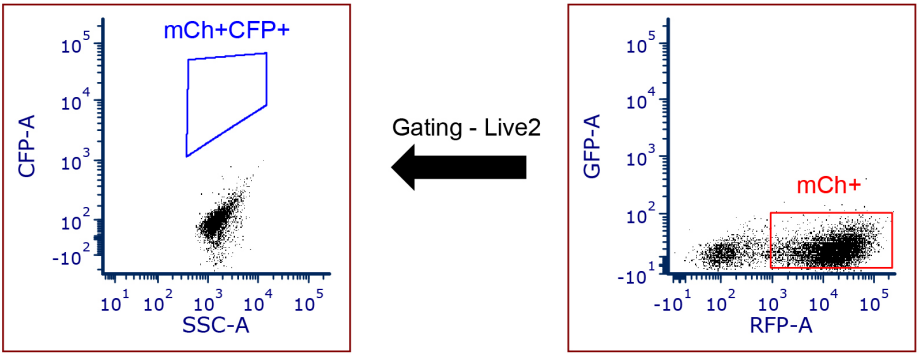

GFP-only control
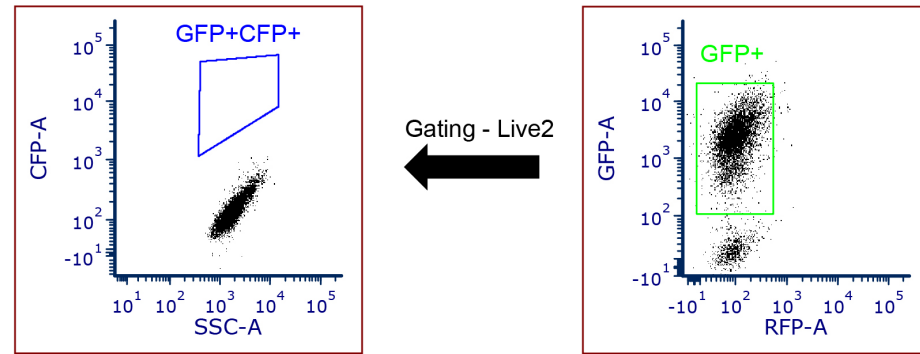

Figure 4. A schematic showing flow cytometry gating strategy. Apply the gating for single and live cells by using forward and side scattering. For the individual fluorescence (GFP and mCherry), gating was applied based on the negative control, GFP-only control, and mCherryonly controls. The gates for selecting GFP and mCherryFP double-positive cells should be at least one fold above the upper edge of the signals from the GFP-only and mCherryFP-only cells.

c. Apply the gating for GFP and mCherryFP double-positive cells in the sampling group and then look for the CFP positive cells in this population. The gate for CFP should be established by using a CFP-negative control cell expressing GFP plus mCherryFP but not CFP. In our experience, this threshold can also be set by using cells carrying the reporter (with mCherryFP marker) and PQC-XIG (with GFP marker). The gating should be set up so 
that it is at least two folds higher than the border of the main population of the CFP signal generated from the CFP-negative cells.

d. The same set of gatings should be universally applied to all sample groups. The presence of CFP fluorescence indicates the occurrence of in/del events and thereby successful genome editing (See examples in Figure 5). The non-specific (ns) scramble-sequence gRNA-GFP (Figure 5) reflects the background signals of spontaneously generated CFP events by non-specific gene editing.
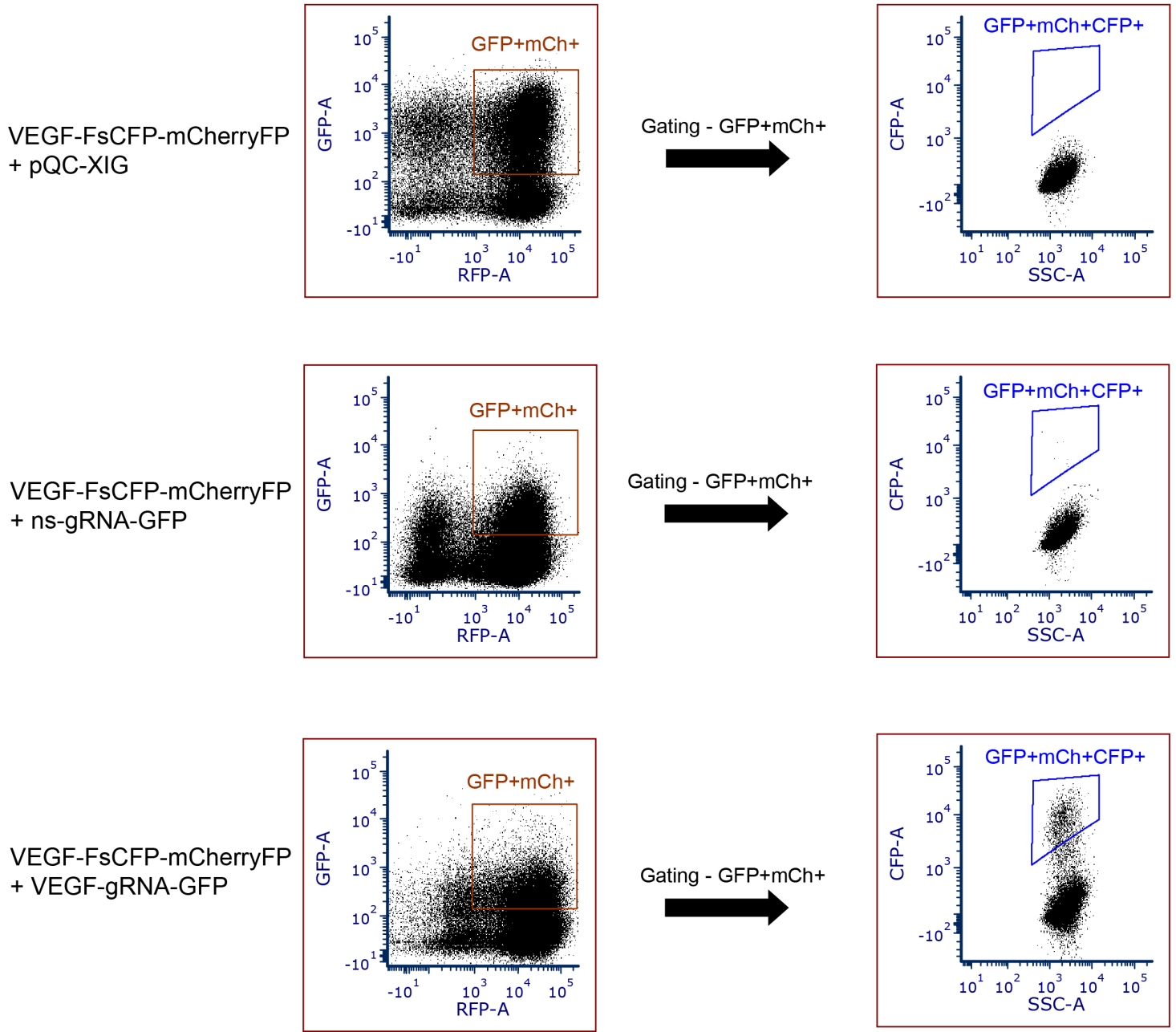

Figure 5. Examples of data to depict the CFP-positive events using this reporter tool in HEK293T cells. Data showing the absence of CFP fluorescence (FsCFP-reporter with target sequence derived from human VEGF gene + pQC-XIG); background CFP fluorescence due to spontaneous mutageneis (reporter + non-specific (ns) gRNA); and presence of CFP-positive events occurred by in/del events (reporter + VEGF gRNA). GFP+mCh+ indicates the gating of double-positive cells (GFP and mCherry). GFP $+m \mathrm{Ch}+\mathrm{CFP}+$ indicates the presence of CFP+ positive events in the double-positive cells (GFP and mCherry). 
e. The number or the percentage of CFP events among the double positives in each cell population, which is the readout of the genome editing efficiency, can be calculated using MS-Excel or the build-in function of the software (FCS Express 6 in this case) .

8. Analysis of genome sequence cell colonies

If desirable, single-cell colonies can be produced by dilution and spreading of the sorted cells in 96-well plates for DNA analysis. See Figure 6 for a summary of all steps in the test.

\begin{tabular}{|c|c|}
\hline \multicolumn{2}{|c|}{ Molecular Cloning for Reporter Plasmid } \\
\hline \multicolumn{2}{|c|}{$\begin{array}{l}\text { Molecular Cloning for Reporter Plasmid } \\
\text { Annealing }\end{array}$} \\
\hline & $\downarrow \quad 1$ day \\
\hline Insertion & $\begin{array}{l}\text { Clone the target sequence into } \\
\text { pQC-FsCFP-mCherryFP }\end{array}$ \\
\hline & $\downarrow$ \\
\hline Sequenc & $\begin{array}{l}\text { Validate the indentify of the repoter } \\
\text { plasmid }\end{array}$ \\
\hline & $1-3$ days \\
\hline & $\downarrow$ \\
\hline Preparation of cel & earrying the reporter \\
\hline Packagir & $\begin{array}{l}\text { Constrution of FsCFP-mCherryFP } \\
\text { viral particles in HEK293T cells }\end{array}$ \\
\hline & $\downarrow$ \\
\hline Infection & $\begin{array}{l}\text { Incubation of target cells with } \\
\text { viral particles }\end{array}$ \\
\hline & $\downarrow$ \\
\hline Sorting & $\begin{array}{l}\text { FACS to isolate cells } \\
\text { expressing CherryFP }\end{array}$ \\
\hline & $\downarrow \quad 1$ day \\
\hline Expansion & $\begin{array}{l}\text { Stabilization and passaging of } \\
\text { CherryFP-positive cell line }\end{array}$ \\
\hline & 3-7 days \\
\hline Preclearing & $\begin{array}{l}\text { (OPTIONAL) FACS to remove } \\
\text { spontaneous CFP-positive cells }\end{array}$ \\
\hline & $\downarrow \quad 1$ day \\
\hline Reporter assay & \\
\hline Infection & $\begin{array}{l}\text { Introduction of viral particle for } \\
\text { Cas9-gRNA with GFP marker }\end{array}$ \\
\hline & $\downarrow$ \\
\hline Incubation & Time to introduction of In-del events \\
\hline & $\downarrow \gamma>=1$ day \\
\hline $\begin{array}{l}\text { Flow } \\
\text { Cytometry }\end{array}$ & $\begin{array}{l}\text { Detection of CFP-psositive events in } \\
\text { cell populations }\end{array}$ \\
\hline & $\begin{array}{l}\downarrow \\
\text { Analysis }\end{array}$ \\
\hline
\end{tabular}

Figure 6. Diagram representing workflow and estimated times to establish a cell line carrying the FsCFP-mCherryFP reporter and perform the genomic editing assay (with Cas9 as an example) 
Notes:

1. If the target sequence contains an internal START codon, care should be taken to ensure that it would not lead to in-frame translation of the CFP. If this is the case, one additional nucleotide can be inserted after the PAM site (preferably after the premature STOP codon) to further shift the reading frame.

2. This method is sensitive for low-frequency in/del events too. We have tested this by mutating two central nucleotides in the targeting $g R N A$, which is expected to reduce the targeting efficiency significantly. When this mutated gRNA is co-expressed along with the reporter, a much reduced yet still statistically significant CFP signal was detected in the cells expressing both GFP and mCherryFP.

3. Spontaneous mutagenesis, such as those resulted from base excision, may also lead to the reactivation of the CFP signal. However, in our experience, the magnitude of such spontaneous events (as demonstrated cells with the reporter and $p Q C-X I G$ vector) are at least one order lower than the non-specific action of Cas9 supplemented with a non-specific gRNA. Therefore, in our experience, it is safe to use the cells carrying the reporter (with mCherryFP marker) and the $P Q C-X I G$ vector (with GFP marker) to set up the detection gate for CFP. However, if a lower background is desired for setting up the CFP detection gate, a cell that only expressing GFP and mCherryFP and not carrying any coding sequence of CFP can be used as the control.

4. The gate for CFP detection can be moved up or down for a few (1-5) folds, as long as the threshold is above the border of the main population in CFP-negative control cells (with GFP and mCherryFP double-positive) and as long as the gate is applied universally to all sampling groups, the differences between each group will still exist.

\section{Acknowledgment}

We thank the flow cytometry core facility at the Sylvester Comprehensive Cancer Center for providing the service. We also thank the funding resources-NIGMS/NIH, R01\#GM107333; DoD (CDMRP), Idea Award, PC140622. The salary of the authors and the cost of experiments are supported in part by these funding resources. These funding bodies were not directly involved in any part of the design of study, the collection, analysis, or interpretation of the data, or writing of the manuscript. This protocol was adapted from this published work (Kumar et al., 2019).

\section{Competing interests}

The authors declare that they have no competing financial interests. 


\section{References}

1. Christian, M., Cermak, T., Doyle, E. L., Schmidt, C., Zhang, F., Hummel, A., Bogdanove, A. J. and Voytas, D. F. (2010). Targeting DNA double-strand breaks with TAL effector nucleases. Genetics 186(2): 757-761.

2. Cong, L., Ran, F. A., Cox, D., Lin, S., Barretto, R., Habib, N., Hsu, P. D., Wu, X., Jiang, W., Marraffini, L. A. and Zhang, F. (2013). Multiplex genome engineering using CRISPR/Cas systems. Science 339(6121): 819-823.

3. Epinat, J. C., Arnould, S., Chames, P., Rochaix, P., Desfontaines, D., Puzin, C., Patin, A., Zanghellini, A., Paques, F. and Lacroix, E. (2003). A novel engineered meganuclease induces homologous recombination in yeast and mammalian cells. Nucleic Acids Res 31(11): 2952-2962.

4. Jinek, M., Chylinski, K., Fonfara, I., Hauer, M., Doudna, J. A. and Charpentier, E. (2012). $\underline{A}$ programmable dual-RNA-guided DNA endonuclease in adaptive bacterial immunity. Science 337(6096): 816-821.

5. Kim, Y. G., Cha, J. and Chandrasegaran, S. (1996). Hybrid restriction enzymes: zinc finger fusions to Fok I cleavage domain. Proc Natl Acad Sci U S A 93(3): 1156-1160.

6. Kumar, A., Birnbaum, M. D., Moorthy, B. T., Singh, J., Palovcak, A., Patel, D. M. and Zhang, F. (2019). Insertion/deletion-activated frame-shift fluorescence protein is a sensitive reporter for genomic DNA editing. BMC Genomics 20(1): 609.

7. Maeder, M. L. and Gersbach, C. A. (2016). Genome-editing technologies for gene and cell therapy. Mol Ther 24(3): 430-446.

8. Sander, J. D. and Joung, J. K. (2014). CRISPR-Cas systems for editing, regulating and targeting genomes. Nat Biotechnol 32(4): 347-355. 